\title{
Swarm Intelligence Techniques to improve lifetime of Wireless Sensor Networks
}

Brahm Prakash Dahiya

I. K. G. Punjab Technical University

India

brahmprakasd@gmail.com

Shewta Rani, Pramjeet Singh

Maharaja Ranjeet Singh Punjab Technical University

India

garg_shavy@yahoo.com

param2009@yahoo.com

ABSTRACT: Wireless Sensor networks (WSNs) is collection of various sensor devices to capture the environment conditions. Node deployment, limited energy capacity, location of sensor devices, Quality of Services (QoS) and data aggregation are the critical design challenges in WSNs. To overcome these design challenges in WSNs, many techniques have proposed. Swarm Intelligence (SI) is one of the most appropriate technique to overcome the design challenges in WSNs.SI shows a current computational and behavioral similitude for taking care of disseminated issues that initially took its motivation from the biological illustrations gave by social insects like ants, termites, honey bees, wasp. Here, we will discuss many SI techniques such that Ant Colony Optimization (ACO), Elephant swarm Optimization (ESO), Hnee based optimization (HBO) and Particle Swarm Optimization (PSO) to make network energy efficient and improve the WSNs lifespan.

Keywords: Wireless Sensor Networks (WSNs), Swarm Intelligence (SI), Ant Colony Optimization (ACO) and Particle Swarm Optimization (PSO)

DOI: $10.6025 / \mathrm{stj} / 2018 / 7 / 2 / 63-75$

Received: 2 May 2018, Revised 29 May 2018, Accepted 5 June 2018

(C) 2018 DLINE. All Rights Reserved

\section{Introduction}

Wireless sensor networks (WSNs) are progressively utilized as a part of utilizations, for example, military, medicinal services, natural, organic, basic health observation, and state built forecasting. With the colossal headway in the arena of implanted PC and WSNS device innovation, WSN's comprise of billions of network sensor nodes which are equipped for detecting, activating and handing-off the gathered data. The capability of Wireless Sensor Network is out and out progressive. This innovation will influence all parts of our lives sooner rather than later.

Signals and Telecommunication Journal Volume 7 Number 2 September 2018 


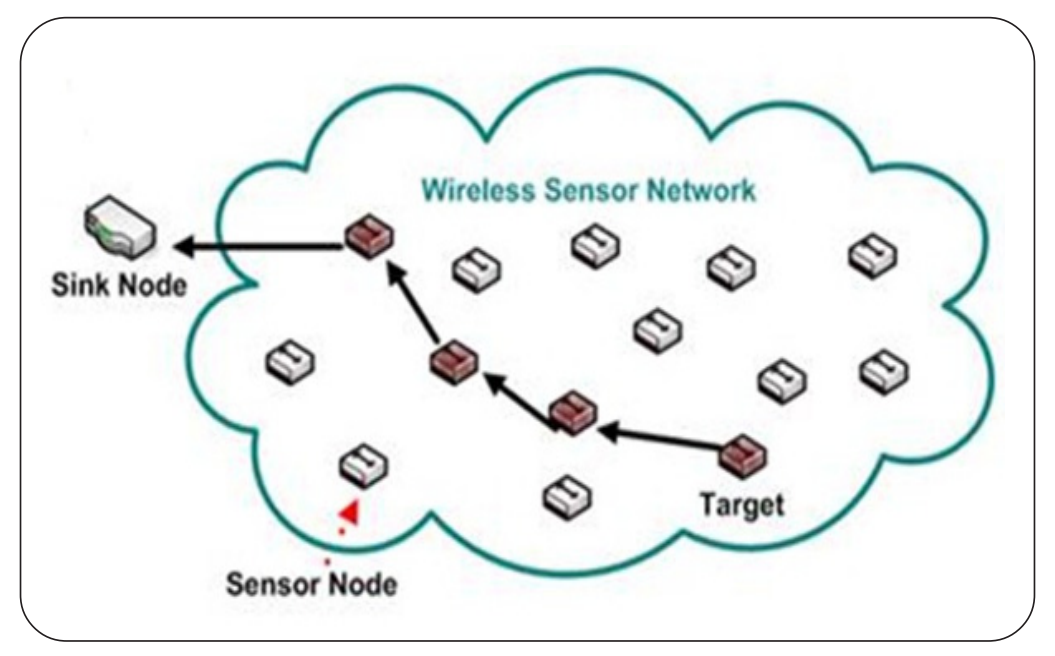

Figure 1. Wireless Sensor Networks

The role of a sensor node in a sensor region is to identify occurrences, perform neighborhood information preparing, and transmit crude or potentially processed information. Sink node is only a base station which assumes a crucial part in remote condition and it acts like an appropriated controller. Base station is essential for the accompanying reasons: sensor nodes are inclined to down, better accumulation of information and gives the backup if the main node is not properly working or failure [1].

\section{Design Challenges in WSNs}

WSNs are influenced by many challenging factors as summarized below:

\section{- Node Deployment:}

Node deployment is big challenge for WSNs lifetime. Deployment implies situating an effective network sensor arranges in certifiable domain. WSNs nodes can be conveyed either by putting in a steady progression in a sensor field. Arrangement of sensor node organize is a work escalated and bulky movement as we don't have effect over the nature of remote correspondence and additionally this existing reality places straining on sensor hubs by insensitive amid communication. Node deployment is very critical issue in WSNs [2,3].

1. Amid the organization of WSNs, Sensor node momentary because of energy utilization (consumption) caused either by emblematic battery release, for the reason that of short circuits is a main issue which may rapid incorrect sensor readings. Likewise issue influencing sink hubs ought to be recognized to limit information misfortune, as sink hub store and forward the gathered information hence go about as an entryway.

2. Another normal issue of certifiable sending of sensor hubs is Low information yield, which implies organize isn't conveying adequate measure of data as required.

3. Because of various simultaneous transmission endeavors made by the sensor hub, organization may bring about WSNs congestion issue. Additional dispute is the physical obstruction in reality because of which two close hubs will be unable to speak through other.

4. Self-Configuration (SF) of WSNs without mortal mediation is requisite for arbitrary organization of sensor nodes.

\section{- Limited Energy Capacity:}

WSNs nodes have battery power. Energy poses a big challenge for lifetime of network. At the point when the energy of a sensor achieves a specific limit, the sensor wound up plainly broken and did not have the capacity to work appropriately. In this way, It is a noteworthy effect on the WSNs performance $[4,5,6]$. The constraints regularly connected with sensor arrange configuration is that sensor nodes work with restricted vitality saves. Ordinarily, they are powered through batteries, which must be either

\begin{tabular}{lllllll}
\hline 64 & Signals and Telecommunication Journal & Volume & 7 & Number & 2 & September \\
\hline
\end{tabular}


displaced or recharged when drained. A few nodes just are disposed of once their energy source is drained. Regardless of whether the battery can be energized or not fundamentally effects the methodology connected to energy utilization. Therefore the critical outline challenge for a WSN is energy efficiency.

\section{- Location of Sensors:}

The requirement for node localization is a standout amongst the most essential difficulties while considering mobility. Localization in WSN implies assessing the position or spatial directions of sensors. Significance of Localization emerges from the need to label the detected information and connect occasions with their area of event. Location data of a sensor node can be gotten by utilizing GPS. In any case, introducing GPS in each sensor isn't reasonable solution. This is on the grounds that sensor nodes are installed in a vast number. Installing GPS at each node will increase the cost and network size. GPS spend power, which will influence the system lifetime [7].

\section{- Quality of Service:}

Data should be conveyed inside a specific time-frame from the period it is sensed. Something else, the information will be pointless. In any case, it is troublesome in light of the way that the framework topology may change persistently and the open state information for steering is naturally free. Sensor network systems ought to be furnished with the required measure of information transmission so it can fulfill an irrelevant compulsory QoS. In WSNs traffic is irregular in sensor arrange since the information is gathered from various cluster node to a sink node [8].

\section{- Data Aggregation:}

Data Aggregation is characterized by way of collection of the information from various sensors with the objective to dispose of repetitive communication and evaluating the coveted response about the sensors condition. Sensor systems are uncertain in natural surroundings. Confident information might be inaccessible or costly to get the WSNs nodes and the many of nodes that are really reacting and furthermore it is hard to discover finish and all information of the neighboring sensor nodes. Enabling couple of nodes to transmit information straight forwardly to the base station (BS) which have fewer communication of information to the base station to reduce energy consumption. Take out redundant information transmitted from sensor nodes by utilizing meta-information arrangements as in Sensor Protocols for Information via Negotiation (SPIN) protocol. To enhancing WSNs aggregation which implies sending in part aggregated values instead of crude esteems, in this way decreasing power consumption [9].

\section{Swarm Intelligence (SI)}

Swarm Intelligence (SI) shows a current computational and behavioral similitude for taking care of disseminated issues that initially took its motivation from the biological illustrations gave by social insects like ants, termites, honey bees, wasp. Nonetheless, these days SI alludes all the for the most part to the investigation of the aggregate behavior of multi-segment frameworks that arrange utilizing decentralized controls and self-association. From an engineering perspective, SI accentuates the bottom-up plan of autonomous frameworks that can demonstrate adaptable, dynamic, and scalable practices. The SI systems incorporate other well-known systems, for example, Ant Colony Optimization (ACO), Particle Swarm Optimization (PSO), Hnee based optimization and Elephant swarm Optimization. ACO is performed to enhance the lifetime of WSNs with energy efficiency. Every wireless sensor node is demonstrated as a mock ant and automatic routing is demonstrated as an ant searching. The ant indication is introduced at the point when a power saving station from the source to base station is protected. To make enhancement in lifetime of WSNs by optimal path selection based on residual energy. Particle swarm improvement (PSO) approach is a swarm intelligence propelled advancement system that has a place with a classification of artificial intelligence. PSO approach begins from the investigation of the nature of the behavior of predacious birds. The essential guideline of PSO approach is that each bird is inattentive as a particle, and the enhanced outcome compares to the position of the particles in the inquiry space [10]. In every cycle step, the particles are updated by following the accompanying two extremes: one is the best position of the neighborhood arrangement and another is the best position of the comprehensive optimal solution. Through their learning background and the trading of all particles data, it decides the following stage of the flight speed and direction, and it step by step advances toward the optimal solution. Accordingly, the presentation of PSO approach can generally enhance the WSNs execution as far as load adjust vitality utilization and so forth. Honey bee improvement (HBO) approach is utilized as a part of this work to perform viable grouping of nodes based on the distance from the diet sources. In addition, it considers the fitness of the food source which is only the energy accessible in the nodes. Here, two types of honey bees are considered to be specific utilized foragers and jobless foragers. Among the jobless foragers two sorts of honey bees in particular scout honey bees which are in duty of searching the food and spectators which are in responsive for watching the hive are utilized as a part 
of this work [11]. Elephant Based Swarm Optimization (EBSO) way to deal with enhance WSNs lifetime. Elephants are social warm blooded creatures [12] and show propelled knowledge [13]. Elephants are frequently found to be present in a "liquid splitting combination" social condition [14]. Elephants portrayed by their great memory, their tendency to exist together and make due inside a cluster [15], socially detailed amid testing circumstances such as movement and when the resources are panic. Elephants identified a magnanimous conduct which empowers them to develop and is the mystery of their life span. Remembering advancement and survivability the more seasoned elephants separate or isolate from the group. Elephants by environment are more grounded defensive of their more youthful age [15][16][17]. Every one of these highlights displayed has affected the creators to join such conduct in WSNs to enhance network throughput.

\subsection{Swarm Intelligence Techniques for the Deployment Problem of WSN}

WSNs development is a troublesome activity which generally impacts the throughput of WSNs as far as cost, quality WSNs lifespan. WSNs design and deployment is to locate the ideal area, where the sensor nodes could be put. SI techniques are profoundly valuable in tackling the plan and developments issues of WSNs. The preeminent goal of sensor node deployment or positioning algorithm is to reduce energy consumption, scope and availability. In view of the applications, the design and deployment of sensors can be static or dynamic. If there should be an occurrence of static sensor establish, the topology design or the organization will be deterministic, then again for dynamic sensor arrange, topology is made non-deterministically. For both the topology creation design, clustering is notable and perceived methods to raise an associated order and to reduce the energy consumption, in this manner improve the network lifespan. In this section different types of SI optimization techniques will introduce to resolve the design and deployment problem of WSNs $[18,19]$.

\subsubsection{ACO based Optimization}

A hexagonal grid-based and ant colony sensor deployment approach proposed, which utilize the technique for hexagonal grid plot and ant colony approach to place sensor nodes to the suitable places of WSNs. The calculation incorporates both deterministic and self-sorting out arrangements in a bound together structure. Furthermore, it not just ensures the greatest scope of sensor arrangement, yet in addition decreases sensor node moving distance [20]. Easi Design: an enhanced ACO approach for sending in genuine sensor arrange framework. An alteration in the convergence approach and the ant state move governs of the common ACO. Making an allowance for the feasibility issues, we outline the difficulty prevention and the route establishment cost tradeoff systems to guarantee that Easi Design cans effort effectively. It depends on two sorts of useful issues: upgrading the routing hops and avoiding obstacles. Right off the bat give another pheromone refreshing principle which considers the quantity of sensors as well as the directing expense in the built arrangement, and after that we outline an impediment location segment to manage the ants to circumvent the obstacles. After the correlations Easi Design accomplishes preferable execution over the traditional ant colony approach [21]. To determine sensor node deployments issue in WSNs an ant colony optimization (ACO) algorithm proposed. It is a proficient deployment plan can decrease the arrangement cost and upgrade the identification capacity of the WSNs. Likewise, it can upgrade the quality of checking in remote sensor arranges by expanding the coverage area. It gives a characteristic and inborn method for investigation of scan space for multiple knapsack problems (MKP). We show the sending issue as the numerous backpack issues. The proposed deployment plans to draw out the network lifetime, while guaranteeing complete scope of the network area [22]. Enhanced version of ACO with three classes of ant transitions (ACO-TCAT) is proposed to diminish lesser rate arrangements and narrow the looking scope of the approach. It settles minimum-cost and connectivity guaranteed grid coverage (MCGC) issues for the accomplishment of WSNs. It epitomizes three classes of ant transitions. The decent varieties of modules of ant transitions is connected to minor arrangements and limited the looking scope of the calculation and furthermore enhances the nature of the arrangement space and raise the seeking speed detectably. In addition, it manages diverse sizes of networks. Moreover, the availability of the framework is ensured for various sink areas and the framework cost is reduced [23]. A novel deployment algorithm, ACO-Greedy is proposed. It tackled the issue of grid-based coverage with low-cost and connectivity-guarantee (GCLC). It depends on the ACO with greedy movement approach, which can rapidly entire the full scope, and notably diminish the organization cost. Furthermore, ACOGreedy can powerfully modify the sensing, detecting and communication range to lighten the energy gap issue and enhance the WSNs lifespan [24]. A novel ant colony optimization (ACO) is proposed for blindness deficiency maintaining a strategic distance from in WSNs. In first stage, a non-blind aggregate based association instrument is intended to decrease deployment cost. Second stage, a non-blind load-balancing approach is intended to accomplish stack adjusting on request in the genuine sense. At long last, reenactments demonstrate that our discoveries fundamentally beat the best in deployment cost as far as sending expense and network lifetime expectancy [25]. A revised version Ant Colony Optimization based LEACH (RA-LEACH) has been proposed for sensor nodes outline and deployment. Cluster Heads (CHs) played out the accompanying parameters like the proportion of the residual energy at every node with its unique energy and the proportion of the distance of the node 
from the Base Station (BS) with the distance of the most distant node. Based on the demonstration, RA-LEACH shows preferred execution over LEACH as for the quantity of alive nodes and energy utilization [26]. A novel algorithm is mix of ant colony optimization algorithm and local heuristic approach. It illuminates the minimum cost reliability obliged sensor node deployment issue (MCRC-SDP). In result comes about on 24 issues cases with various operational parameters showed the viability of the proposed approach in discovering brilliant solutions for the issue. Results likewise demonstrate that the quality of the acquired solutions by the proposed ACO approach is better than that of solutions got by a Greedy Heuristic by over $20 \%$ all things considered [27]. A novel ACO-GD, in view of the idea of ant colony optimization (ACO) algorithm and a thought of gradient diffusion (GD). A preparatory approach was outlined and proposed in this paper before ACO-GD. It is a non-uniform gradient diffusion approach and can be utilized to tackle the energy holes issues and expand the network lifetime. The preparatory approach was then coordinated with the ACO approach. The result comes about demonstrate that our ACO-GD calculation performs better effectiveness for the cost of scope, arrange lifetime, and the quantity of nodes [28].

\subsubsection{Hnee based Optimization}

HBO approach is utilized to defeat basic coverage issue in WSN [29]. Utilizing a similar approach provides solution of Q-k coverage issues in WSNs [30]. After solution of coverage issue, it enhanced WSNs lifespan; an artificial honey bee colony approach is introduced [31]. The scenario is focused for presentations, where the PoI is identified as earlier. Preceding the premise of probabilistic detection model (PDM) to extend coverage scope an advance dynamic deployment of static and portable WSNs nodes introduced [32]. According to PoI is secured by various probabilities. On the off chance that when a single PoI is interfaced to singular sensor, the coverage and the system unwavering quality will be extensively low. Subsequently covering objective coverage is the dire requirement for dynamical deployment. This is proficient by discussed probabilistic identification demonstrate through $\mathrm{ABC}$ approach [33].

\subsubsection{PSO based Design and Deployment}

Fuzzy adaptive particle swarm optimization is mix of PSO and fuzzy system. It is actualized to progressively modify the latency mass to enhance the throughput of the PSO. Three standard capacities utilized for testing the throughput of the fuzzy adaptive PSO. Comparative performance conducted using simulator both the fuzzy adaptive PSO and the PSO with a linearly diminishing latency weight. The outcomes additionally outline that PSO with a fuzzy system tuning its inertia weight enhance performance and improve lifetime of WSNs [34] [35]. The cooperative particle swarm optimizer (CPSO) approach is proposed. It is utilizing cooperative behavior to altogether enhance the performance of the existing approach. This is accomplished by utilizing numerous swarms to enhance distinctive parts of the arrangement vector cooperatively. Use of the new PSO approach on a few benchmark advancement issues demonstrates a checked change in performance over the conventional PSO [36]. A VFCPSO approach is proposed. It is called the VFCPSO. It is an enhanced co-evolutionary PSO approach for dynamic deployment. In the planned approach, CPSO is received to actualize global searching of optimal deployment vectors in co-developmental way, virtual power is utilized to coordinate the refreshing of particles towards the better positions. The result comes about outline the extraordinary execution of VFCPSO approach, i.e., VFCPSO is more advanced than the VF, PSO and VFPSO calculation as far as powerful scope region and computation time and the throughput of the VFCPSO approach is almost steady as the quantity of WSNs increments [37]. An improved PSO is introduced to solve sensor node deployment issue and enhance WSNs lifetime. The change of PSO is powerful and accomplishes a superior execution than VF algorithm [38]. An adaptive particle swarm optimizer is proposed to enhance the WSNs lifetimes. The adaptive criterion is affixed on singular level. Since the critical constant ${ }^{\circ}$ is chosen by the accuracy prerequisite to fitness, it is all the more effectively to be chosen for various issues [39]. An enhanced discrete particle swarm optimization (DPSO) is proposed in view of target coverage control technique for WSNs. It is actualized under two procedures: deployment arranging and movement control. Results delineate that in development control, the enhanced DPSO which is anything but difficult to deal with, is of superior which is no not as much as conventional discrete issue solver [40]. The reader arrangement is the primary issue for the most difficult RFID network planning (RNP), and should be sensibly explained to work the huge scale RFID frameworks in an ideal mold. An enhanced Particle Swarm Algorithm is proposed (SAPSO) to take care of this issue, which can limit the position change of unique and new particles in the cycle procedure and quicken the merging velocity of the calculation. The reproduction comes about demonstrate that the tended to SA-PSO approach is better than the traditional algorithm [41]. Particle Swarm Optimization (PSO) approach is proposed to solve Voronai issue i.e. suffered from deployment strategy. So to locate the position of the node with less number of dead nodes another system is recommended in this exploration work which utilizes Voronai PSO which may bring about discovering ideal coverage region for sensors and lessen number of dead nodes [42]. An ABC-PSO vertical handover decision approach is proposed for single and multi-attribute issue, individually, with the goals of minimizing the normal aggregate cost and limiting the average quantity of handovers. The proposed algorithm has a lesser add up to cost and average quantity of handovers than the Technique for Order Preference by Similarity to Ideal Solution (TOPSIS) approach [43]. 


\subsection{Energy Efficient Node Clustering}

Battery limitation is one of the real issues in sensor nodes. Once the WSNs nodes are set in remote areas, revive and change of battery isn't a simple undertaking. Subsequently energy efficiency is one of the vital objectives to enhance the lifetime of WSNs and availability of the sensor network. Different SI based energy efficient node clustering techniques are used for saving energy of sensor nodes [44].

\subsubsection{ACO based Clustering Optimization}

An efficient routing approach is proposed for substantial scale group based WSNs. The approach utilizes two routing levels. In the primary level, cluster members individuals send information specifically to their cluster head. In the second level, the cluster heads utilize ant colony optimization (ACO) approach, which is an organically roused worldview for improvement approach, to discover a route to the base station. The delay of the approach is limited by utilizing the ant colony optimization algorithm alongside clustering [45]. A novel energy efficient Ants colony optimization clustering (ACO-C) approach is proposed in WSNs. It used to choose optimal cluster head determination and powerfully transformed the distance between the cluster heads to distribute clusters all through the network. Results demonstrate the adequacy of proposed approach over other clustering protocols for example, LEACH, LEACH-C and PSO-C [46]. An inter-cluster Ant Colony Optimization algorithm (IC-ACO) is proposed based on ACO algorithm. It avoids wastage of redundant data sent by the sensors to base station. It is more energy efficient as compare to existing protocols [47]. An energy efficient and load balanced ant colony optimization based hierarchical data gathering method (ACOHC) is proposed. The deployment zone is partitioned into ideal KOPT number of clusters utilizing $\mathrm{K}$-mean. The cluster head selection is performed using ACO. It reduced data delay and improves WSNs lifetimes [48]. Intercluster Ant Colony Optimization algorithm is proposed for transfer data packets in WSNs. It reduced packets wastage during the routing times. In result the inter cluster ACO data aggregation is best as compare to the rendezvous Leach [49].

\subsubsection{Hnee based Clustering Optimization}

Energy distributed clustering Hnee based clustering is proposed to suitable cluster head selection. Utilizing energy and distance factor cluster head choice performed. It works to enhance WSNs lifetime and efficient energy utilization. As per test comes about it is more effective than LEACH and Clustered Multiple Routing (UCR) approach. It additionally maintained a strategic distance from information repetition and work to choose ideal route selection [50]. Unequally Clustered Multiple Routing (UCMR) is proposed utilizing Honey Bee Optimization (HBO). In simulation result demonstrated that energy utilization of node for each round in proposed network is minimum as compare to LEACH, UCR, and UCMR. So this approach is basic and in addition utilizes different parameters, for example, energy, no of neighbor sensor node and position of sensor nodes to choose $\mathrm{CH}$ which improve the WSNs lifespan [51]. Honey Bee Optimization is proposed to reduce the energy consumption and find best path from source to destination. It improved network lifetime of WSNs. The proposed approach is to broaden the lifetime of a system and throughput and it conveys preferable performance over existing strategy as far as the energy effectiveness, end to end connectivity, and scalability. Cluster formation and selection is performed using biologically inspired artificial bee colony approach. Additionally energy level of nodes is calculated by the intelligent agent and also optimal route is selected by the intelligent agent [52].

\subsubsection{PSO based Clustering Optimization}

Particles swarm optimization (PSO) approach is proposed to cluster head formation and selection. It uses two main slave cluster head to save energy and improve network lifetime. It is shown best result as compared than LEACH and PSO [53]. A PSO inspired approach acquainted with resolves clustering and routing issue in WSNs. It is working in two stages: first stage takes a shot at find appropriate cluster head and second stage tackles the issue of the between inter-cluster communication utilizing the best route selection. It enhanced packets delivery rate at both base station and Cluster head. It likewise enhanced WSNs lifetime and coverage. It connected in GPS for area discovery [54]. Energy efficient PSO based routing algorithm with Mobile Sink (EPMS) is presented for WSNs. Virtual clustering technique merged with PSO approach to enhance the WSNs lifetimes. Cluster head election performed based on left energy and node location information. The controlling procedure of mobile sink node depends on the gathering of information from different cluster heads. According to simulation results it shown better performance as compare to existing routing algorithms for WSNs [55]. Optimized QoS-based Clustering with Multipath Routing Protocol (OQoS-CMRP) is introduced for WSNs to reduce energy consumption in base station coverage area using the modified PSO based clustering approach to formulate and selection suitable cluster head. It avoids energy holes issue. It accomplishes better load balancing by powerfully picking exchange way from subset of best-case ways to transmit information. It provides better performance as compare to EE-LEACH and EPSO-CEO [56]. ABC-PSO vertical handover decision approach is proposed for single and multi-property circumstances, respectively, with the objectives of minimizing the normal aggregate cost and minimizing the normal number of handovers. We have connected the AHP strategy to figure the weight of each QoS factor 
in the cost function, and received the proposed calculation under MIH to give link intelligence and other related system data to upper layers. It also avoids unusual handover. It is better as compare to TOPSIS. Based on lower total cost and lower average number of handover, it is better than the TOPSIS algorithm [57].

\subsection{Localization in WSNs}

Localization is a standout amongst the most significant subjects in light of the fact that the area data is ordinarily valuable for routing, organization, deployment, rescue operation and target following [1]. Subsequently, location estimation is a huge specialized challenge for the analysts. What's more, restriction is one of the key strategies in WSN. In this paper we will examine different restriction based SI procedures [58].

\subsubsection{ACO based Localization in WSNs}

A node localization approach is proposed based on mobile beacon. Utilize one mobile beacon is replaced by virtual beacons rather than original condition, lessening the cost of localization. This work has a smaller amount of virtual beacon points and shorter visiting way length contrasted with Hilbert approach, so sparing energy drops. Acquire the ideal arrangement of virtual beacon points for every node by the ideal sifting strategy, so we can additionally enhance the localization exactness [58]. A Bees Optimization Algorithm (BOA) is proposed for localizing the nodes of the WSNs. BOA is populace based searching approach that plays out an area look joined with random pursuit. It is enlivened by natural foraging behavior of honey bees. The summation of the squared range mistake between the node and the stays is utilized as the target capacity to be limited in this work [59]. A novel deployment approach, ACO-Greedy approach is proposed to take care of issue of grid-based coverage with low-cost and connectivity-guarantee (GCLC). It depends on the ACO with greedy migration method, which can rapidly entire the full coverage, and particularly diminish the deployment cost factors. It is likewise changing the detecting/correspondence range to ease the energy holes issue and enhanced the WSNs lifetime [60]. ACO with Mobile Anchor Positioning (ACO-MAP) is introduced for localization in WSNs to solve location exactness of Mobile Anchor Positioning with Mobile Anchor and Neighbor (MAP-M\&N). This approach reduces localization error fundamentally by $96.9 \%$ on a normal when contrasted with MAP-M\&N approach. Subsequently clearly ACO-MAP developmental approach limits the mistake in limitation much superior to utilizing MAP-M\&N approach [61].

\subsubsection{PSO based Localization in WSNs}

Particle swarm search localization approach is exhibited utilizing constraint-based WSNs. In first stage, it characterized constraints domain of an obscure. At that point the locations which satisfy particular criteria are sought out by PSO approach and the looking outcomes inside the constraint domain are noted. Finally, the obscure nodes localization can be gotten by ascertaining the normal recording results. As is appeared in the comparison comes about, CPL has solid robustness, and contrasting and typical plans, for example, least square technique (LS), and CPL's situating accurateness can enhance half when the going fault is 35\% [62]. Other ways to implement Particle Swarm Optimization (PSO) way to deal with perform fine tune and get exact estimation of the areas. The proposed approach is partitioned into two stages. At the primary stage an enhanced variant of DV distance composed with multi modification was utilized to give coarse introductory area measurements. It takes care of issue of localization, for example, flip vagueness, aggregate interpretation and error propagation. To assess the execution of the approach, numerical recreations executed and the outcomes were contrasted and comparable separation based techniques, to be specific one-stage simulated annealing (SA), trilateration and simulated annealing (TSA) and semi-definite programming localization (SDP) [63]. Particle swarm optimal is proposed based on quantum to enhance the location accurateness of sensor nodes in WSNs. It has quicker convergence, shorter computation time, and higher clarification exactness. It can finish the optimal situating of WSNs viably and rapidly, and to a great extent enhance the situating exactness of WSNs, which assumes an imperative part in broadening the life time of WSNs [64]. The idea of various relocation variations of Biogeography-Based Optimization (BBO) approach and Particle Swarm Optimization (PSO) for conveyed ideal optimal localization of randomly deployed sensors nodes. Biogeography is aggregate learning of land assignment of natural life forms. PSO models had just quick convergence yet less develop [65]. Opposite side PSO is attainable to take care of multidimensional optimization issue as a result of its brisk convergence and direct request for computing assets. In this work, the underlying pursuit search space is characterized by bounding box strategy and a refinement stage is advanced to rectify the error because of flip uncertainty. Further, the control of iterative mistake propagation and energy utilization is potential and huge bearings [66]. This new calculation utilizes a streamlining approach the conventional PSO for localization, and utilizations a localization approach maximum likelihood estimation (MLE) to keep beginning extent and the region iterative procedure of PSO confinement process. A MLE-PSO indoor restriction calculation in view of Received signal strength indicator (RSSI) is proposed to enhance the limitation exactness and dynamic execution. The proposed MLE-PSO takes full favorable position of the high exactness of MLE while extending mistake is little and in addition the solidness of PSO while running blunder is enormous, so the MLE-PSO

Signals and Telecommunication Journal Volume 7 Number 2 September 2018 
calculation displays higher precision contrasted and customary MLE calculation and PSO calculation. At the interim, MLE-PSO calculation needs fewer circumstances of cycles and less populace of molecule swarm, which demonstrates that MLE-PSO has better powerful execution [67].

\subsection{Data Aggregation in WSNs}

Data Aggregation is characterized as the way toward collected the information from various sensor nodes with the objective to dispose of repetitive communication and evaluating the coveted answer about the sensors condition. In this section we will introduce various SI techniques based on data aggregation in WSNs [68].

\subsubsection{ACO based on Data Aggregation in WSNs and other SI Techniques}

An ant colony optimization approach is proposed in view of information collection to defeat the blemishes of existing ant-based data-centric routing algorithms. Trial comes about demonstrate that the proposed calculation performs considerably more adequately than other customary techniques, for example, Ant, DD and GIT. The proposed approach has brought down directing overhead, transmitting overhead and normal delay [68]. Data Aggregation Ant Colony Algorithms (DAACA) works with three stages: introduction, data communication and procedures on pheromones. In the transmit stage, every sensor node evaluates the rest of the energy and the measure of pheromones of neighbor node to figure the probabilities for powerfully choosing the next sensor node. After specific rounds of transmissions, the pheromones alterations are performed, which take the upsides of both worldwide and neighborhood merits for dissipating or saving pheromones. Four unique pheromones modification techniques are intended to accomplish the worldwide network lifetime, in particular Basic-DAACA, ES-DAACA, MM-DAACA and ACS-DAACA. Contrasted and some other data aggregation approach, DAACA demonstrates higher prevalence by and large level of sensor node, energy efficiency and improve network lifetime [69]. Ant-colony aggregation based on distributed approach is proposed to reduce energy consumption and reduces network traffic by remove information repetition in the message sending process. It gives an inherent method for investigating the look space to streamline settings for optimal data aggregation. It reduces energy consumption and enhance network lifetime in WSNs [70]. Another probabilistic collection convention in view of ACO and Genetic Algorithm (GA) mixture approach is proposed. The Multi-Objective Steiner Tree (MOST) is characterized as the ideal structure for information conglomeration, which can be investigated and every now and again misused amid the routing procedure. The route choice is powerfully achieved by computing the pheromone and the heuristic. This versatile planning arrangement has the capacity of decreasing the transmission delay and enhancing aggregation probability. Through the reenactment comes about, the proposed convention shows better exhibitions when compared with other existing conventions [71]. An Inter-cluster ACO approach is proposed to lessen the energy utilization issue and furthermore totals and transmits the information in destructive way by utilizing the ACO. Origin $\mathrm{CH}$ conveys collected data to sink and cluster heads impart information to the root $\mathrm{CH}$ specifically or by implication in view of the created root by ant colony improvement. The similar examination has demonstrated that the proposed ant colony improvement based iLEACH beats over the accessible conventions as far as the different quality measurements [72].

\subsection{Quality of Services}

Data should be conveyed inside a specific time-frame from the period it is sensed. Something else, the information will be pointless. In any case, it is troublesome in light of the way that the framework topology may change persistently and the open state information for steering is naturally free.

\subsubsection{ACO based Quality of Services Optimization and other SI Techniques}

A novel Quality of Service (QoS) routing methodology is proposed in light of Ant Colony Optimization (ACO) to decrease throughout complexity and energy utilization of the QoS routing techniues. ACO approach utilizing aggregate cleverness of artificial ants as intelligent agents is exceptionally proper to tackle the combinatorial enhancement issues in a completely dispersed manner. An enhanced version of ACO approach is acquainted with unravels Delay Constraint Maximum Energy Residual Ratio (DCMERR) QoS directing issue of WSNs. [73]. An energy-effective QoS routing approach is proposed to speeds up the convergence of ant colony algorithm by using stateless non-deterministic geographic forwarding (SNGF). SNGF approach is utilized to influence ant determination to be more meaningful. The pheromone is characterized as a mix of connection load and bandwidth delay, at that point, with a specific end goal to adjust the energy utilization of WSNs, node energy is utilized as the control factor of the ant colony approach [74]. A trust QoS routing approach is introduced in light of the Particle Swarm Optimization (PSO) to discover the route with most maximum residual energy and unwavering quality keeping in mind the end goal to boost network use and enhance its throughput. Contrasted with existing routing algorithms in WSNs, this new approach has given best results [75]. A hybrid Ant Colony Optimization (ACO)/Particle Swarm Optimization (PSO) approach is proposed make more effective the multicast tree. It settle delay, packets loss rate, data transfer capacity, and postpone jitter issues. The

\begin{tabular}{lllllll}
\hline 70 & Signals and Telecommunication Journal Volume & 7 & Number 2 & September 2018
\end{tabular} 
proposed approach developed the multicast tree designs all the more sensibly to such an extent that the tree designs fulfil the QoS requirements, as well as tries to limit the tree cost [76]. Optimized QoS-based Clustering with Multipath Routing Protocol (OQoS-CMRP) approach is acquaint with improve WSNs performance by applying the Modified Particle Swarm Optimization (PSO)- based clustering approach to from sink node to cluster head to resolve energy holes issues. The Single Sink-All Destination approach is utilized to discover close ideal multi-hop correspondence way from sink to sensors for choosing the next hop neighbor nodes. The Round-robin Paths Selection approach is utilized for exchanging information to sink. It likewise diminishes transmission postponement and correspondence overhead based on guaranteeing the result of the whole system [77].

\section{Conclusion and Future Work}

Wireless Sensor networks (WSNs) is collection of various sensor devices to capture the environment conditions. Node deployment, limited energy capacity, location of sensor devices, Quality of Services (QoS) and data aggregation are the critical design challenges in WSNs. To overcome these design challenges in WSNs, many techniques have proposed. Swarm Intelligence (SI) is one of the most appropriate technique to overcome the design challenges in WSNs.SI shows a current computational and behavioral similitude for taking care of disseminated issues that initially took its motivation from the biological illustrations gave by social insects like ants, termites, honey bees, wasp. In above sections, we discussed many SI techniques such that Ant Colony Optimization (ACO), Elephant swarm Optimization (ESO), Hnee based optimization (HBO) and Particle Swarm Optimization (PSO) to make network energy efficient and improve the WSNs lifespan. ACO, HBO and PSO based approaches have applied to resolve the sensor nodes deployment issue and helpful in energy utilization and extend WSNs lifespan. In next section SI techniques are merged with clustering algorithms and gave best results. These SI techniques are more results oriented, when applied on location, data aggregation and Quality of Services Challenges. In this survey the author discussed all SI techniques on different sides. In future work, this survey will more beneficial to select suitable technique for WSNs design challenges and based on the existing SI techniques, we can design more energy efficient algorithm to extend WSNs lifetime.

\section{References}

[1] Lu, Weijie., Bai, Donglin. (2016), Energy efficient distributed lifetime optimizing scheme for wireless sensor networks, Transactions of Tianjin University Journal, 22 (1) 11-18.

[2] Pooja, Singh., S. (2016). Improved O-LEACH protocol: A clustering based approach in wireless microsensor network, In: IEEE 10th International Conference on Intelligent Systems and Control (ISCO) 1-4.

[3] Bari, Ataul., Arunita, Jaekel., Bandyopadhyay, Subir. (2016), Clustering strategies for improving the lifetime of two-tiered sensor networks, Elsevier Computer Communication Journal, 3, p. 3451-3459.

[4] Sarma, N. V. S. N, Gopi, Mahesh. (2014), Energy Efficient Clustering using Jumper Firefly Algorithm in Wireless Sensor Networks, International Journal of Engineering Trends and Technology (IJETT), 10 (11) 525-532.

[5] Chen, Hua., Luo, Min., Sun, Baolin. (2014), Energy Efficient Based Maximal Lifetime Routing in Wireless Sensor Networks, International Journal of Future Generation Communication and Networking, 7 (3) 69-76.

[6] Singh, Lovedeep., Dayal, Priyanka. (2014), Lifetime Improvement using Adaptive Modulation in Wireless Sensor Network, International Journal of Computer Networks and Wireless Communications, 4 (5) 340-344.

[7] Luo, X., Ji, X., Park, M. (2010), Location privacy against traffic analysis attacks in wireless sensor networks, In: International Conference on Information Science and Applications, p. 1-6.

[8] Tran Cong Hung, Nguyen Hong Quan. (2014). A proposal for improve the lifetime of wireless Sensor Network, IEEE International Journal of Computer Networks \& Communications (IJCNC), 6 (5) 59-69.

[9] Fasolo. E. (2007), In-network Aggregation Techniques for Wireless Sensor Networks: A survey, IEEE Wireless Communications, 14 (2) 70-87.

[10] Wang, J., Ren, X., Shen, Y., Liu, S. (2010), A remote wireless sensor network for water quality monitoring, International Conference on Innovative Computing and Communication, p.7-12.

[11] Liang, Y., Yu, H. (2005), PSO-based energy efficient gathering in sensor networks, Lecture Notes in Computer Science, p. 362-369. 
[12] Ado Adamou Abba Ari, Blaise OmerYenke, Labraoui, Nabila., Damakoa, Irepran., Gueroui, Abdelhak. (2016). A power efficient cluster based routing algorithm for wireless sensor networks: Honey bees swarm intelligence based approach, Elsevier, Journal of Network and ComputerApplications, vol. 69, p.77-97.

[13] Wilson, E. O. (2000), Sociobiology: The New Synthesis. 25th Anniversary Editions. The Belknap Press of Harvard University Press Cambridge, Massachusetts and London, England.

[14] Krebs, J. R., Davies, N. B. (1993). An Introduction to Behavioral Ecology. Third Edition. Blackwell Publishing, Oxford, UK.

[15] Elizabeth, A., Archie, Cynthia, J., Moss, Susan, C., Alberts. (2006). The ties that bind: genetic relatedness predicts the fission and fusion of social groups in wild African elephants, Proc. R. Soc. B 273, p. 513-522.

[16] Elizabeth, A., Archie, Patrick, I., Chiyo. (2012). Elephant behavior and conservation: social relationships, the effects of poaching, and genetic tools for management, Molecular Ecology, vol. 21, p. 765-778.

[17] Adams, Rachael. (2013). Social Behavior and Communication in Elephants- It's true! Elephants don't forget!, Available at: http://www.wildlifepictures-online.com/ elephant-communication.html.

[18] Wyatt, T. D. (2003). Pheromones and Animal behavior - Communication by Smell and Taste. Cambridge University Press. $\mathrm{UK}$.

[19] Younis, O., Krunz, M., Ramasubramanian, S. (2006). Node Clustering in Wireless Sensor Networks: Recent Developments and Deployment Challenges, IEEE Network, vol. 20 (3) 20-25.

[20] Xiao, J., Han, S., Zhang, Y., Xu, G. (2010). Hexagonal grid-based sensor deployment algorithm, In: Proceedings Control Decision Conference, p. 4342-4346.

[21] Li, D., Liu, W., Cui, L. (2010). Easi Design: An improved ant colony algorithm for sensor deployment in real sensor network system, IEEE Global Communications Conference, p.1-5.

[22] Liao, Wen-Hwa., Kao, Yucheng., Wu, Ru-Ting. (2016). Ant colony optimization based sensor deployment protocol for wireless sensor networks. Expert Systems with Applications, vol. 38. p. 6599-6605.

[23] Xuxun, L. (2012). Sensor Deployment of Wireless Sensor Networks Based on Ant Colony Optimization with Three Classes of Ant Transitions, IEEE Communications Letters, vol. 16, p.1604-1607.

[24] Liu, He, D. (2014). Ant colony optimization with greedy migration mechanism for nod deployment in wireless sensor networks, Journal of Net- work and Computer Applications, vol. 39, p. 310 -318.

[25] Huang, G., Chen, D., Liu, X. (2015). A node deployment strategy for blindness avoiding in wireless sensor networks, IEEE Communications Letters, 19 (6) 1005-1008.

[26] Liu, X. (2015). A deployment strategy for multiple types of requirements in wireless sensor networks, IEEE Transactions on Cybernetics, 45 (10) 2364-2376.

[27] Sakai, K., Sun, M. T., Ku, W. S., Lai, T. H., Vasilakos, A.V. (2015). A framework for the optimal k-Coverage deployment patterns of wireless sensors, IEEE Sensors Journal, 15 (12) 7273-7283.

[28] Shun-Miao Zhang, Degen Huang., Shu-Chuan Chu, Tien-Wen Sung., Tsu-Yang Wu. (2017). An Adaptive ACO-Based Node Deployment Algorithm in Wireless Sensor Networks, Journal of Internet Technology, 18 (5) 1193-1202.

[29] Mini, S., Udgata, S. K., Sabat, S. L. (2013). Sensor deployment in 3-D terrain using artificial bee colony algorithm, In: Proceedings Swarm, Evolutionary, and Memetic Computing, p.424-431.

[30] Mini, S., Udgata, S. K., Sabat. (2011). Artificial bee colony based sensor deployment algorithm for target coverage problem in 3-D terrain, In: Proceedings Distributed Computing International Technology, p. 313-324.

[31] Mini, S., Siba, K., Udgata, Samrat, L., Sabat. (2014). Sensor Deployment and Scheduling for Target Coverage Problem in Wireless Sensor Networks, IEEE Sensors Journal, 14 (3).

[32] Chaudhary, M., Pujari, A. K. (2009). Q-coverage problem in wireless sensor networks, In: Proceedings International Conference Distribution Computer Networks, p. 325-330.

[33] Ozturk, C., Karaboga, D., Gorkemli, B. (2011). Probabilistic dynamic deployment of wireless sensor networks by artificial bee colony algorithm, Sensors, 11 (6) 6056-6065. 
[34] Shi, Yuhui., Eberhart, R. C. (2001). Fuzzy adaptive particle swarm optimization, In: Proceedings of the 2001 Congress on Evolutionary Computation (IEEE Cat. No.01TH8546), Seoul, vo1, p. 101-106.

[35] Eberhart, Shi, Yuhui. (2001). Particle swarm optimization: developments, applications and resources, In: Proceedings of the 2001 Congress on Evolutionary Computation (IEEE Cat. No.01TH8546), Seoul, vol 1, p. 81-86.

[36] Van den Bergh, F., Engelbrecht, A. P. (2004). A Cooperative approach to particle swarm optimization, IEEE Transactions on Evolutionary Computation, 8 (3) 225-239.

[37] Wang, X., Wang, S., Ma, J. J. (2007). An improved co-evolutionary particle swarm optimization for wireless sensor networks with dynamic deployment, Sensors, vol.7, p. 354-370.

[38] Li, Z., Lei, L. Sensor Node Deployment in Wireless Sensor Networks Based on Improved Particle Swarm Optimization, In: Proceedings. IEEE International Conference on Applied Superconductivity and Electromagnetic Devices, p. 215-217.

[39] Kumar, Vinod., Khanna, Pranav., Bisht, Sanjay. (2012). Adaptive PSO based Algorithm for Optimal WSN Deployment in 3 Dimensional Terrain, National Conference on Advancement of Technologies - Information Systems \& Computer Networks, $\mathrm{p}$. $1-6$.

[40] Du, H., Pan, Q., Pan, Q., Yao, Y., Lv, Q. (2014). An improved Particle Swarm Optimization-Based Coverage control method for wireless sensor network, Advances in Swarm Intelligence Lecture Notes in Computer Science, vol. 87, p. 114-124.

[41] Tao, Ming., Huang, Shuqiang., Li, Yang., Yan, Min., Zhou, Yuyu. (2014). SA-PSO based optimizing reader deployment in large-scale RFID Systems, Journal of Network and Computer Applications, vol. 52, p. 90-100.

[42] Kaur, Harjeet., Kautish, Sandeep. (2016). An Implementation of Wireless Sensor Network Using Voronoi_PSO (Particle Swarm Optimization), International Journal for Research in Applied Science \& Engineering Technology, 4 (11) 361-368.

[43] Shidrokh, Goudarzi., Hassan, Wan Haslina., Anisi, Mohammad Hossein., Soleymani, Ahmad., Sookhak, Mehdi., Khurram, Muhammad., Khan, Abdalla Hashim, Aisha-Hassan., Zareei, Mahdi (2017). ABC-PSO for vertical handover in heterogeneous wireless networks, Neurocomputing, vol. 256, p. 63-81.

[44] Ashouri, M., Yousefi, H., Basiri, J., Hemmatyar, A. M. A., Movaghar (2015). A. PDC: Prediction-based data-aware clustering in wireless sensor networks, Journal of Parallel and Distributed Computing. vol. 81, p. 24-35.

[45] Salehpour, A., Mirmobin, B., Afzali-kusha, A. (2008). An Energy Efficient Routing Protocol fo Cluster-based Wireless Sensor Networks Using Ant Colony Optimization, IEEE Proceedings of the 5th International Conference on Innovations in Information Technology, p. 455-459.

[46] Ziyadi, M., Yasami, K., Abolhassani, B. (2009). Adaptive Clustering for Energy Efficient Wireless Sensor Networks Based on ant Colony Optimization, IEEE Proceedings of the 7th Annual Communication Networks and Services Research Conference, p. 330-334.

[47] Kim, J. Y., Sharma, T., Kumar, B., Tomar, G. S., Berry, K., Lee, W. H. (2014). Inter cluster ant colony optimization algorithm for wireless sensor network in dense environment, International Journal of Distributed Sensor Networks.

[48] Mondal, S., Ghosh, S., Biswas, U. (2016). ACOHC: Ant colony optimization based hierarchical clustering in wireless sensor network, 2016 International Conference on Emerging Technological Trends (ICETT), Kollam, p. 1-7.

[49] Ghotra, A. (2017). Optimizing Inter Cluster Ant Colony Optimization Data Aggregation Algorithm with Rendezvous Nodes and Mobile Sink, Wireless Sensor Network,vol. 9, p.16-24.

[50] Kaur, Kiranpreet., Singh, Harjit. (2015). Cluster Head Selection using Honey Bee Optimization in Wireless Sensor Network, International Journal of Advanced Research in Computer and Communication Engineering, 4 (5) 358-363.

[51] Rani, Nisha., Singh, Jaswinder. (2016). Honey Bee Optimization based Zone Divided Wireless Sensor Network, International Journal of Engineering Research \& Technology, 5 (4)73-77.

[52] Selvi, M., Nandhini, C., Thangaramya, K., Kulothungan, K., Kannan, A. (2017). HBO based clustering and energy optimized routing algorithm for WSN, In: Eighth International Conference on Advanced Computing (ICoAC), Chennai, p. 89-92.

[53] Yu, Hu., Xiaohui, Wang. (2011). PSO-based Energy-balanced Double Cluster-heads Clustering Routing for wireless sensor networks, In: Procedia Engineering, vol.15, p. 3073-3077.

[54] Riham, S. Y., Elhabyan, Mustapha. C. E., Yagoub. (2015). Two-tier particle swarm optimization protocol for clustering and 
routing in wireless sensor network, Journal of Network and Computer Applications, vol 52, p. 116-128.

[55] Wang, Jin., Cao, Yiquan., Li, Bin., Kim, Hye-jin., Lee, Sungyoung. (2017). Particle swarm optimization based clustering algorithm with mobile sink for WSNs, Future Generation Computer Systems, vol. 76, p. 452-457.

[56] Deepa, O., Suguna, J. (2017). An optimized QoS-based clustering with multipath routing protocol for Wireless Sensor Networks, Journal of King Saud University - Computer and Information Sciences.

[57] Goudarzi, Shidrokh. (2017). Wan Haslina Hassan, Mohammad Hossein Anisi, Ahmad Soleymani, Mehdi Sookhak, Muhammad Khurram Khan, Aisha-Hassan Abdalla Hashim, Mahdi Zareei, ABC-PSO for vertical handover in heterogeneous wireless networks, In: Neurocomputing, vol. 256, p. 63-81.

[58] Qin, F., Wei, C., Kezhong, L. (2010). Node Localization with a Mobile Beacon Based on Ant Colony Algorithm in Wireless Sensor Networks, In: Proceedings of International Conference on Communications and Mobile Computing, p. 303-307.

[59] Moussa, A., El-Sheimy, N. (2010). Localization of wireless sensor network using bees optimization algorithm, In: Proceedings of IEEE International Symposium on Signal Processing and Information Technology, p.478-481.

[60] Liu, X., He, D. (2014). Ant colony optimization with greedy migration mechanism for node deployment in wireless sensor networks, Journal of Network and Computer Applications. Vol. 39, p. 310-318.

[61] Sivakumar, S., Venkatesan, R. (2016). Error Minimization in Localization of Wireless Sensor Networks using Ant Colony Optimization, International Journal of Computer Applications, 145 (8) 15-21.

[62] Shu-wang, Z., Ying-long, W., Qiang, G., Nuo, W. (2010). Constraint-based sensor network nodes particle swarm search localization algorithm, The 2010 14th International Conference on Computer Supported Cooperative Work in Design, Shanghai, China, p. 448-451.

[63] Namin, P. H., Tinati, M. A. (2011). Node localization using Particle Swarm Optimization, 2011 Seventh International Conference on Intelligent Sensors, Sensor Networks and Information Processing, Adelaide, p. 288-293.

[64] Jian-Bin, Y., Wen-Bo, X. (2011). Research on the Node Localization Based on Quantum Particle Swarm Optimal Algorithm for WSNs, 2011 10th International Symposium on Distributed Computing and Applications to Business, Engineering and Science, Wuxi, p. 309-313.

[65] Singh, S., Shivangna., Mittal, E. (2013). Range Based Wireless Sensor Node Localization Using PSO and BBO and Its Variants, 2013 International Conference on Communication Systems and Network Technologies, Gwalior, p. 309-315.

[66] Li, Dan., Xian bin Wen. (2015). An Improved PSO Algorithm for Distributed Localization in Wireless Sensor Networks, International Journal of Distributed Sensor Networks, vol. 11, p. 970-272.

[67] Zhao, C., Wang, B. (2017). A MLE-PSO indoor localization algorithm based on RSSI, 36th Chinese Control Conference (CCC), Dalian, p. 6011-6015.

[68] Wang, Hongpeng., Luo, Neng. (2014). An Improved Ant-Based Algorithm for Data Aggregation in Wireless Sensor Networks, Communications and Mobile Computing, International Conference, vol. 3., p. 239-243.

[69] Lin, Chi., Wu, Guowei., Xia, Feng., Li, Mingchu., Yao, Lin., Pei, Zhongyi. (2012). Energy efficient ant colony algorithms for data aggregation in wireless sensor networks, Journal of Computer and System Sciences, 78 (6)1686-1702.

[70] Xie, M., Shi, H. (2012). Ant-Colony Optimization Based In-Network Data Aggregation in Wireless Sensor Networks, 12th International Symposium on Pervasive Systems, Algorithms and Networks, San Marcos, p. 77-83.

[71] Lu, Y., Comsa, I. S., Kuonen, P., Hirsbrunner, B. (2015). Probabilistic Data Aggregation Protocol Based on ACO-GA Hybrid Approach in Wireless Sensor Networks, 2015 8th IFIP Wireless and Mobile Networking Conference (WMNC), p. 235-238.

[72] Kaur, J., Chopra, V. (2016). Enhanced iLEACH Using ACO Based Intercluster Data Aggregation. In: Behera H., Mohapatra D. (eds) Computational Intelligence in Data Mining - Advances in Intelligent Systems and Computing, vol. 411.

[73] Cai, W., Jin, X., Zhang, Y., Chen, K., Wang, R. (2006). ACO Based QoS Routing Algorithm for Wireless Sensor Networks. In: Ma, J., Jin, H., Yang, L.T., Tsai, J. JP. (eds) Ubiquitous Intelligence and Computing. UIC 2006. Lecture Notes in Computer Science, vol 4159.

[74] Li, Z., Shi, Q. (2013). An QoS Algorithm Based on ACO for Wireless Sensor Network, In: 2013 IEEE 10th International Conference on High Performance Computing and Communications \& 2013 IEEE International Conference on Embedded and 
Ubiquitous Computing, Zhangjiajie, p. 1671-1674.

[75] Wang, J. W., Wei, Y. T. (2013). PSO-Based Trust QoS Routing Algorithm for Wireless Sensor Networks, Applied Mechanics and Materials, Vol. 401-403, p. 1751-1755.

[76] Manoj Kumar Patel, Manas Ranjan Kabat., Chita Ranjan Tripathy. (2014). A hybrid ACO/PSO based algorithm for QoS multicast routing problem, Ain Shams Engineering Journal, 5 (1) 113-120.

[77] Deepa, O., Suguna, J. (2016). An optimized QoS-based clustering with multipath routing protocol for Wireless Sensor Networks, Journal of King Saud University - Computer and Information Sciences. 